\title{
A Comprehensive Transcript Map of the Mouse Gnas Imprinted Complex
}

\author{
Rebecca Holmes, ${ }^{1}$ Christine Williamson, ${ }^{1}$ Jo Peters, ${ }^{1}$ Paul Denny, ${ }^{1}$ \\ RIKEN GER Group ${ }^{3}$ and GSL Members, ${ }^{4,6}$ and Christine Wells ${ }^{2,5}$ \\ ${ }^{1}$ The Mammalian Genetics Unit, Medical Research Council, Harwell OX11 ORD, Oxfordshire, UK; ${ }^{2}$ The Institute of Molecular \\ Biosciences, The University of QLD 4072, Brisbane, Australia; ${ }^{3}$ Laboratory for Genome Exploration Research Group, RIKEN \\ Genomic Sciences Center (GSC), RIKEN Yokohama Institute, Suehiro-cho, Tsurumi-Ku, Yokohama, Kanagawa, 230-0045, \\ Japan; ${ }^{4}$ Genome Science Laboratory, RIKEN, Hirosawa, Wako, Saitama 351-0198, Japan
}

\begin{abstract}
The recent publication of the FANTOM mouse transcriptome has provided a unique opportunity to study the diversity of transcripts arising from a single gene locus. We have focused on the Gnas complex, as imprinting loci themselves provide unique insights into transcriptional regulation. Thirteen full-length cDNAs from the FANTOM2 set were mapped to the Gnas locus. These represented one previously described transcript and 12 putative new transcripts. Of these, eight were found to be differentially expressed from either the maternal or paternal allele. Two clones extended Nespas in the $3^{\prime}$ direction, providing evidence of antisense transcription spanning a 30-kb genomic region from a single allele. The transcripts were summarized into six transcriptional units, Nespas, Nesp, Gnasxl, F7, exon 1A, and Gnas. The resolution of the Gnas transcript map by the FANTOM2 clones revealed a pattern of alternate splicing. In addition to the transcripts described previously as splicing onto exon 2 of Gnas, each new sense transcript had an alternate short 3'UTR independent of Gnas. Both spliced and unspliced variants of the new imprinted sense transcripts were found. Whereas the functional significance of these alternate transcripts is not known, the availability of the FANTOM clones has provided remarkable insights into the repertoire of transcripts in the Gnas complex locus.
\end{abstract}

[Supplemental material is available online at www.genome.org. The sequence of the clones described in this paper have a RIKEN accession no., and will be assigned GenBank nos. once the data are released.]

Distal Chromosome 2 was one of the first imprinted regions identified in mouse (Cattanach and Kirk 1985). Mice with two maternal copies of the region and no paternal copies [MatDp(dist2)] fail to survive for more than a few hours after birth, are small, and hypoactive. In contrast, mice with two paternal copies but no maternal copies [PatDp(dist2)] are hyperactive, edematous, and usually die within a few days of birth. In recent years, the Gnas complex locus has been shown to lie within the region (Williamson et al. 1996) and to be imprinted (Kelsey et al. 1999; Peters et al. 1999). Some features of the PatDp(dist2) and MatDp(dist2) mice are recapitulated in mice with maternal and paternal inheritance, respectively, of a targeted deletion of Gnas exon 2 (Yu et al. 1998), and also in Oed mice (Cattanach et al. 2000), which have a missense mutation in Gnas exon 6 (Skinner et al. 2002). These observations strongly suggest that Gnas contributes to the PatDp(dist2) and MatDp(dist2) phenotypes. Mice with maternal inheritance of the Gnas exon 2 knockout, however, and also those with a missense mutation in Gnas exon 6, are not hyperactive at birth and variably survive, unlike PatDp(dist2) mice, suggesting that these effects are attributable to other imprinted genes or transcripts at the Gnas locus that exclude exon 2 or exon 6 . In addition, mice with paternal inheritance of the Gnas exon 6 missense mutation show postnatal growth

${ }^{5}$ Corresponding author.

${ }^{6}$ Takahiro Arakawa, Piero Carninci, Jun Kawai, and Yoshihide Hayashizaki.

E-MAIL c.wells@imb.uq.edu.au; FAX 61-7-3365488.

Article and publication are at http://www.genome.org/cgi/doi/10.1101/ gr.955503. retardation (Skinner et al. 2002), as do those mice with paternal inheritance of the Gnas exon 2 knockout that survive the neonatal period (Yu et al. 1998). Therefore, the death of MatDp(dist2) within a few hours of birth may also be attributed to other imprinted genes or transcripts at the Gnas locus that exclude exon 2 or exon 6 .

The Gnas locus is extremely transcriptionally complex through the use of alternative first exons, alternative splicing of downstream exons, and antisense transcripts. Four alternative first exons are known-Nesp, Gnasxl, Gnas exon 1a, and Gnas exon 1-each of which splices onto exon 2 of Gnas itself (Peters et al. 1999; Liu et al. 2000). Nesp is expressed exclusively from the maternal allele, and Gnasxl and Gnas exon 1a are oppositely paternally expressed. Although the Gnas exon 1 transcript is mainly bi-allelically expressed, it shows maternal specific expression in some tissues (Yu et al. 1998). Gnas has 13 exons, but transcripts are known that terminate in a neural exon following exon 3 (Crawford et al. 1993). A number of transcripts antisense to Nesp have been described that are collectively called Nespas and may have a regulatory function (Li et al. 2000; Wroe et al. 2000; Williamson et al. 2002). The discovery of new transcripts at the Gnas locus provides candidates for a complete functional analysis of this region and greater understanding of the MatDp(dist2) and PatDp(dist2) phenotypes.

\section{RESULTS}

The FANTOM2 data set were screened for clones lying in the Gnas imprinting cluster. Thirteen putative Gnas clones were identified through the FANTOM2 annotation process, and de- 
fined by the FANTOM2 genome mapper as part of the known Gnas locus (The FANTOM Consortium 2002). Only one of the FANTOM clones had been described previously and corresponded to Gnas exons 2-13 (data not shown). The other 12 FANTOM clones appeared to represent new transcripts in the Gnas imprinting cluster and their genomic location and exon structure are shown in Figure 1. All splicing followed the GT/ AG rule. Supplementary Table 1 summarizes the tissues from which ESTs associated with each transcript originated.

Strand-specific RT-PCR was performed on RNA derived from 15.5-dpc embryos with maternal duplication [MatDp(dist2)] or paternal duplication [PatDp(dist2)] of the imprinting cluster to confirm the existence of the new transcripts and to establish their imprinting status on the sense or antisense strand. The strand-specific reverse transcription primers, together with the PCR primers for each clone are listed in Supplementary Table 2. The integrity of the RNA was confirmed by reverse transcription with oligo dT and amplification with Hprt primers (Fig. 2A). Classification of embryos was confirmed by expression analysis. Therefore expression of maternal-specific Nesp coupled with lack of expression of paternal-specific Gnasxl confirmed the MatDp(dist2) embryos. Conversely, lack of expression of Nesp, together with expression of Gnasxl, confirmed the PatDp(dist2) embryos (Fig. 2B,C).

\section{Extending the Length of Nespas}

FANTOM cDNA clones, D330038P10 of 629 bp and D030028H20 of $3.8 \mathrm{~kb}$ derived from 13-dpc embryonic heart and 9-dpc embryonic whole body libraries respectively, were designated F1 and F2, respectively (Fig. 1). They gave an excellent sequence match at the $5^{\prime}$ end of Nesp. The match to the BAC genomic sequence (accession no. AL593857) was on the complementary strand, and the association of each clone with ESTs previously orientated as antisense to Nesp, suggested that F1 and F2 are derived from antisense transcripts. An imprinted antisense transcript, Nespas, had been identified previously. F1 and F2 were mapped $15 \mathrm{~kb}$ and $1.87 \mathrm{~kb}$, respectively, from the 3 ' end of a Nespas poly (A) signal. Following strand-specific reverse transcription and amplification (see Fig. 1 and Supplementary Table 2), a band of the expected size was amplified in PatDp(dist2) but not MatDp(dist2) (Fig.
2D,E), confirming that F1 and F2 represent antisense transcripts that are paternally expressed. PCR from F1 to exon IV of the Nespas spliced form (Williamson et al. 2002) revealed the presence of a paternal-specific product (Fig 2L). Sequencing identified two novel exons, the first lying between F1 and F2 (nucleotides 82123-82289, AL593857), the second between F2 and Nespas exon V (nucleotides 69172-96104, AL593857) (Fig. 3). Therefore F1 is part of a new alternative noncoding splice variant of Nespas that extends the previously reported length of Nespas (Williamson et al. 2002) by $15.8 \mathrm{~kb}$. We propose that F2 is also part of a Nespas transcript.

\section{Further Transcripts of Nesp}

The remaining 10 FANTOM cDNAs matched the sense strand of the genomic sequence and all showed matches to ESTs on the sense strand as shown in Figure 1. Clone A230089C09, designated F3, from a 15-dpc embryonic head library, consisted of exons 1 and 2 of Nesp, which spliced into the region between Nesp and Gnasxl, so identifying two new exons as shown in Figure 1. As expected, this new transcript showed maternal-specific expression because after hybridization with a PCR product of EST AA955518 it was detected exclusively in the MatDp(dist2) embryo (Fig. 2F). The presence of several bands in Figure 2F suggests there are a number of splice variants. One of these may be clone D9300020N02 of 1969 bp, designated F4, also from a 15-dpc embryonic head library, which is an unspliced cDNA that lies $3^{\prime}$ of Nesp and shows homology to one of the new exons identified in F3 (Fig. 1). F4 also showed maternal-specific expression (Fig. 2G) after hybridization with a PCR product of EST AA955518. No amplification was observed in the MatDp(dist2) sample without reverse transcriptase showing the RNA was not contaminated with genomic DNA and therefore providing proof that the amplification seen in the MatDp(dist2) sample with reverse transcriptase was caused by the presence of a real transcript.

\section{New Transcripts of Gnasxl}

F5 (clone C130027O20), from a 16-dpc embryonic head library, contains the first exon of Gnasxl and is contiguous with the genomic DNA sequence through the splice

Previously identified ESTs
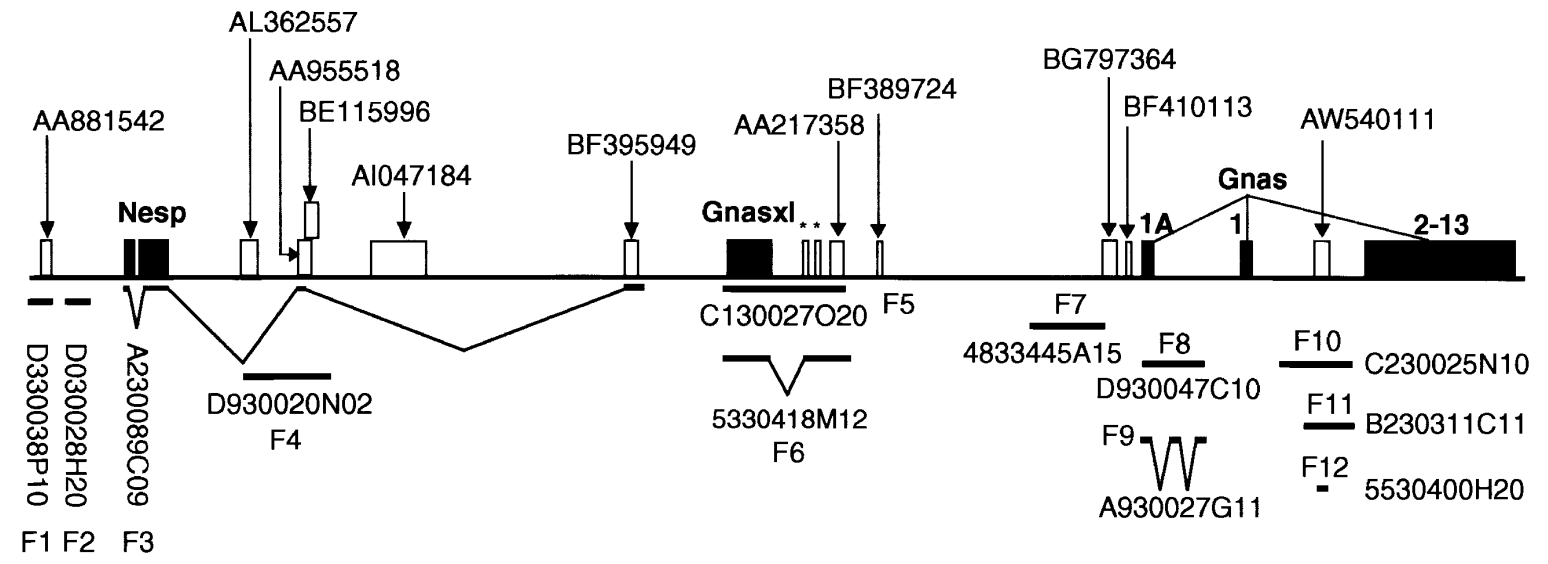

FANTOM2 cDNAs

Figure 1 Relative position of FANTOM2 cDNAs. Previously identified exons (solid boxes) and ESTs (open boxes) are shown above the line and FANTOM2 clones below. Asterisks indicate regions of homology to human A20 and A21 exons. Figure not to scale. 


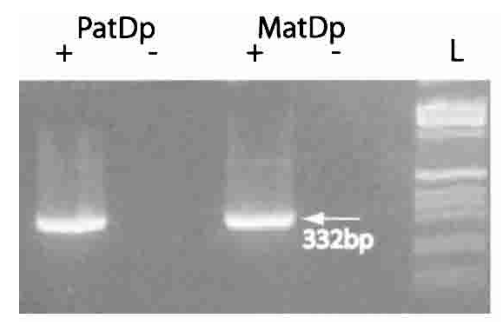

A

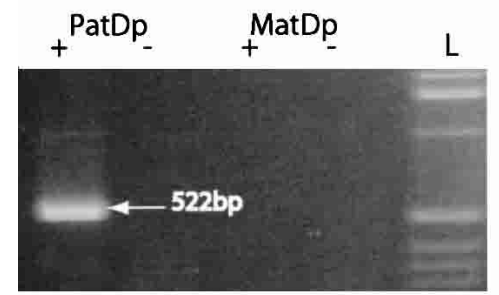

D

F1

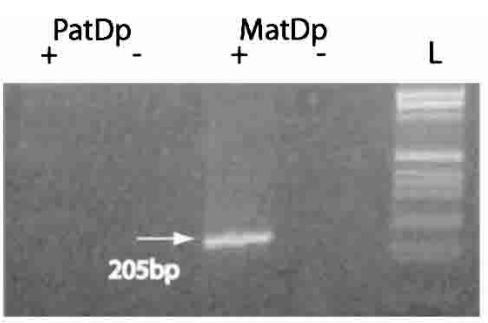

B

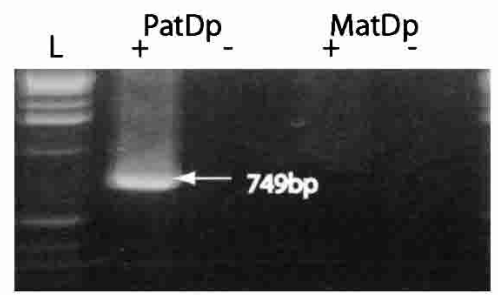

E

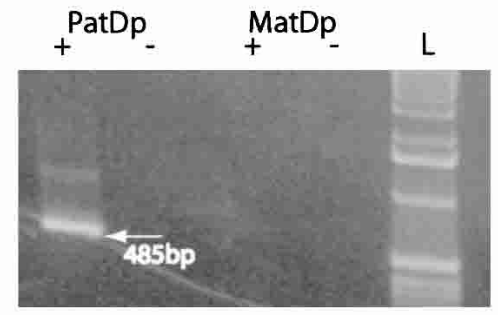

H

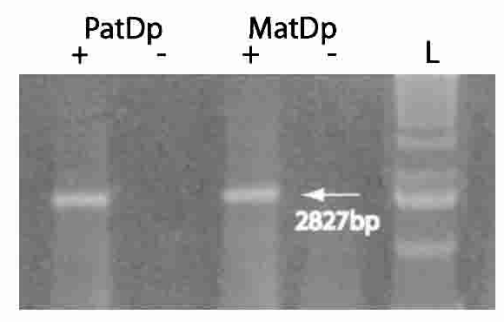

K

F10

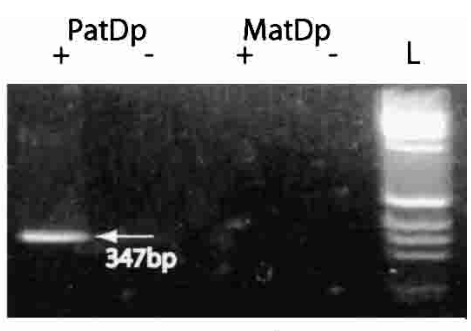

C

Gnasxl

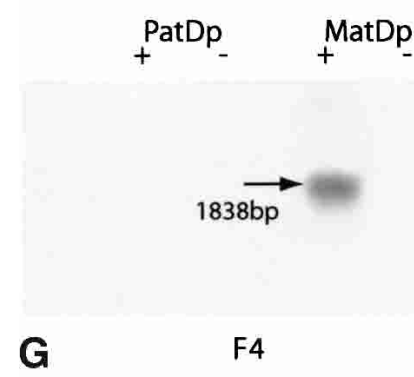

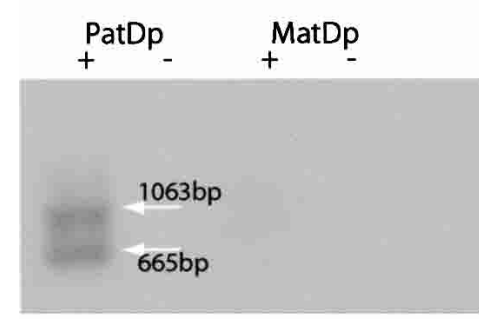

$J$

F8 and F9

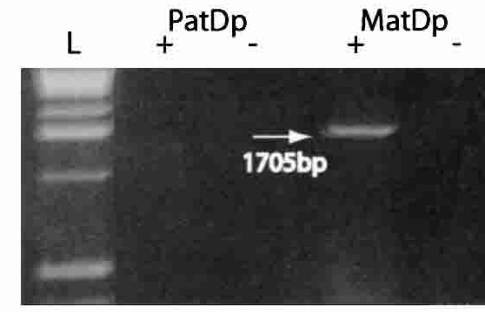

F7

Figure 2 Imprinting status of FANTOM clones F1-F10. Each PCR product is sized with a -kb ladder (Invitrogen). MatDp(dist2) and PatDp(dist2) are abbreviated to MatDp and PatDp, respectively. + and - refers to samples amplified in the presence $(+)$ or absence $(-)$ of reverse transcriptase. Oligo dT-primed cDNA was amplified with: $(A)$ Hprt primers as an amplification control; $(B)$ Nesp primers to confirm the MatDp(dist2) cDNA; and (C) Gnasx/ primers to confirm the PatDp(dist2) cDNA. Strand-specific RT-PCR of FANTOM clones 1-10: (D) paternal expression of F1; (E) paternal expression of $\mathrm{F} 2 ;(F)$ maternal expression of $\mathrm{F} 3 ;(G)$ maternal expression of $\mathrm{F} 4 ;(H)$ paternal expression of F5; $(I)$ maternal expression of $\mathrm{F7}$; $(J)$ paternal expression of F8 and F9; and $(K)$ bi-allelic expression of F10. The PCR products from F3 $(F)$, F4 $(G)$, F7 $(I)$, and F8 $(J)$ were blotted and probed as described in Methods. (L) F1 is part of Nespas. PCR from exon IV of Nespas to F1 reveals a paternal-specific product. The products in $A-C, G, H$, and $L$ were amplified for 25 cycles. The products in $D-F$, I and / were amplified for 30 cycles; the product in $K$ was amplified for 20 cycles. (L) 1-kb Ladder (Invitrogen).

donor site of the exon and through the region that contains sequences homologous to exons A20 and A21, identified in human, as shown in Figure 1. F5 was detected in the PatDp(dist2) embryo but not in the MatDp(dist2) embryo (Fig. 2H), showing the transcript is paternally expressed on the sense strand and therefore represents an alternative form of the Gnasxl transcript. Clone 5330418M12, designated F6, from a pituitary library, is a spliced form of F5 in which Gnasxl splices into the putative mouse A20 exon. Human transcripts in which XLas, the homolog of Gnasxl, splices

\section{Genome Research}




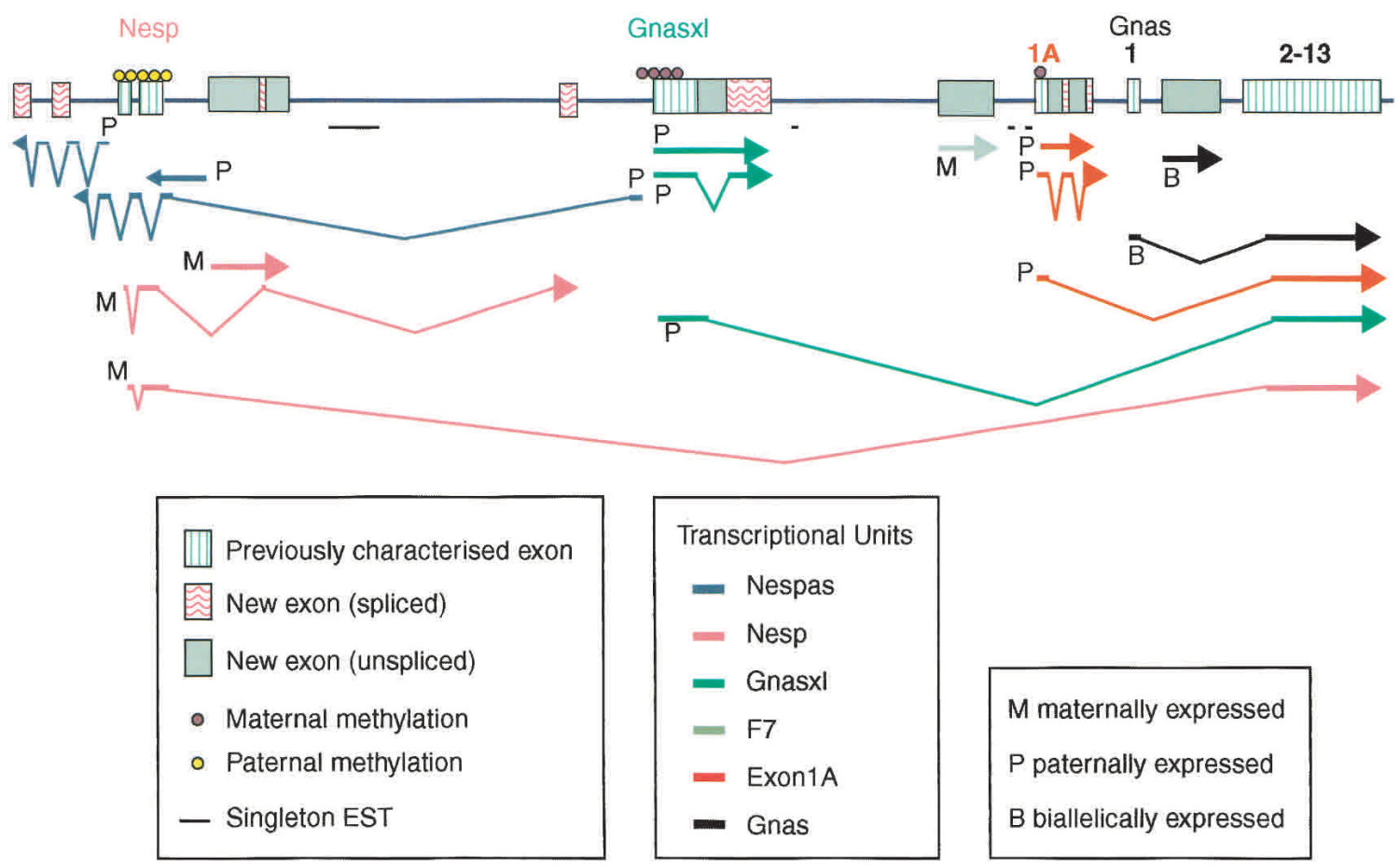

Figure 3 Transcript map of the Gnas complex imprinted locus. Six transcriptional units were defined from this study; these are Nespas, Nesp, Gnasxl, F7, exon 1A, and Gnas. Each transcriptional unit is associated with a differentially methylated promoter, and consists of several splice variants. F7 is the exception, with unexpected maternal expression indicating a link to the Nesp promoter. The arrows show direction of transcription. Figure is not drawn to scale.

into A20 or A21 had been identified previously (Hayward et al. 1998). F6 may be expressed prenatally and this may account for our inability to detect this spliced form in our expression analysis of whole 15.5-dpc embryos (Fig. 2H).

\section{A New Maternal Transcript}

Clone 4833445A15, F7, from a newborn head library, maps between Gnasxl and exon 1A and lies 2138 bp from the $5^{\prime}$ of exon $1 \mathrm{~A}$ and is expressed in MatDp(dist2) but not PatDp(dist2) (Fig 2L). This is the first maternally expressed transcript to be identified between Gnasxl and exon 1A.

\section{Further Transcripts of Gnas Exon 1A}

Clone D930047C10, F8, from a 15-dpc embryonic head library, starts at exon $1 \mathrm{~A}$, is contiguous with the genomic sequence and extends $1080 \mathrm{bp}$ as far as nucleotide 1535 (accession no. AF152375). F8 showed paternal specific expression (Fig. 2J). Therefore F8 appears to represent an unspliced form of the paternally expressed exon 1a transcript on the sense strand. Clone A930027G11, F9, from a retina library, is a spliced form of F8. Using the primers for F8 a smaller product of $\sim 665 \mathrm{bp}$ was amplified in PatDp(dist2) but not MatDp(dist2) (Fig. 2J) and this would be consistent with the existence of a splice form as shown for F9 in Figure 1.

\section{New Gnas Transcripts}

Clone C230025N10, designated F10, from a newborn cerebellum library, lies in the intron between exon 1 and 2 of Gnas but does not extend into either exon (Fig. 1). The transcript was detected in both MatDp(dist2) and PatDp(dist2) thereby showing that this transcript is bi-allelically expressed (Fig. 2K). Two other FANTOM cDNAs, F11 and F12, (clone numbers B230311C11 from a Chrysolina quadrigemina library and $5530400 \mathrm{H} 20$, from a library of 10-day-old head, respectively) represented shortened forms of F10 and are predicted to be bi-allelically expressed. F10 is consistent with bi-allelic expression of Gnas itself (exons 1-13).

\section{DISCUSSION}

Analysis of the FANTOM full-length cDNA collection has allowed a comprehensive transcript summary of the Gnas complex imprinted locus. The Gnas locus was known to be transcriptionally rich, with multiple ESTs mapped previously to the genomic BAC (Fig. 1). Thirteen full-length FANTOM cDNA clones, representing six transcriptional units (TU), describe novel splice variants of Nespas, Nesp, Gnasxl, exon 1A, and Gnas, as well as a completely novel, maternally expressed transcript $5^{\prime}$ of exon $1 \mathrm{~A}$, designated $\mathrm{F} 7$. We predict that each alternatively spliced transcript will be associated with the differentially methylated promoters described previously (Kelsey et al. 1999; Peters et al. 1999; Liu et al. 2000) for each transcriptional unit (Fig. 3). Although the origin of F7 remains a conundrum, the maternal expression of this transcript predicts an association with the Nesp promoter. We observed a common pattern of alternate splicing. In addition to the previously described transcripts in which the $5^{\prime}$ exons of Nesp, Gnasxl, and exon 1A were spliced onto exon 2 of Gnas, each $5^{\prime}$ exon was spliced onto an alternate $3^{\prime}$ UTR independent of Gnas. Additionally, each of these alternate 3' UTRs were expressed as spliced or unspliced variants. The alternatively spliced messages from each transcriptional unit were mono- 
allelically expressed from the same allele. This emerging pattern of transcription may offer insights into the control or processing of imprinted transcripts, though the functional significance of this observation is as yet unclear.

The start site of Nespas was known to lie $\sim 2 \mathrm{~kb}$ upstream of Gnasxl (Li et al. 2000) in a maternally methylated region and the transcripts extend for more than $15 \mathrm{~kb}$ of genomic sequence (Williamson et al. 2002). Nespas has now been extended farther by almost $16 \mathrm{~kb}$ upstream of Nesp, so that it now covers $>30 \mathrm{~kb}$. It may be of significance that the antisense transcripts at human GNAS also cover $>30 \mathrm{~kb}$ of genomic sequence, starting just upstream of XL $\alpha$ s (homologous with Gnasxl) and extending $19 \mathrm{~kb}$ upstream of NESP (Hayward and Bonthron 2000). Furthermore, Nespas may have a function in heart, for it was shown previously to be expressed strongly there (Wroe et al. 2000), and the F1 clone that extends Nespas came from an embryonic heart library. Nespas, like a number of other imprinted antisense genes, such as Air (Wutz et al. 1997; Lyle et al. 2000), is noncoding, paternally expressed, and extends across a large genomic region. Antisense transcription appears to be a common feature of imprinted regions and there is evidence that it has a role in regulating expression from its sense counterpart (Wutz et al. 1997; Lee 2000; Sleutels et al. 2002).

F10, F11, and F12, like Gnas, were bi-allelically expressed, however, we cannot exclude the possibility that these clones are tissue-specifically imprinted. Gnas itself is bi-allelic in most tissues but is maternally expressed in certain cell types in mouse (Yu et al. 1998) and human (Hayward et al. 1998).

It is difficult to speculate how comprehensive a transcript map the FANTOM2 clones provide. We found no cDNA matches in $122 \mathrm{~kb}$ of BAC sequence upstream of the most 5' clone, F1. There is some mouse EST evidence of transcription in this region; however, it is not known whether these ESTs are part of the Gnas-imprinted complex. No cDNA matches were found in the 3.9-kb region between exon 13 of Gnas and the 3 ' end of the BAC sequence. This is consistent with human studies, where the next gene $3^{\prime}$ of Gnas is TH1, which lies $>70 \mathrm{~kb}$ downstream of GNAS1 and is outside the imprinted region (Bonthron et al. 2000).

Analysis of the FANTOM2 data set has revealed a further level of complexity of the single Gnas locus and indicates the massive increase in coverage and diversity found in the mouse transcriptome. Complex regions of transcription like the Gnas locus provoke our definitions of a gene beyond simple exon sharing. The FANTOM2 consortium coined the phrase "Transcriptional Unit" to define a functional clustering of transcripts. This single gene locus contains at least six imprinted TU, each containing several splice variants. Genetic studies have provided strong evidence that the complex phenotypes associated with the region may be attributable to functional differences between these TU. These data indicate the depth of the mouse transcriptome is more than the number of genes in the genome; diversity of transcripts from a single gene locus is the variable that underlies dynamic gene networks.

\section{METHODS}

\section{FANTOM2 cDNA Clone Analysis}

New clones were identified as part of the FANTOM2 annotation process (FANTOM2 Main Paper) and were mapped to BAC sequence of the region (accession no. AL593857, clone number RP23-439H2).

\section{Distal Chr 2-Duplication/Deficient Mice}

Mice with maternal duplication of distal Chr 2 [MatDp(dist2)] and the reciprocal paternal duplication [PatDp(dist2)] were generated by standard methods of intercrossing reciprocal translocation heterozygotes (Searle and Beechey 1978). The reciprocal translocation $\mathrm{T}(2 ; 8) 26 \mathrm{H}$ was used as described previously (Williamson et al. 1996). MatDp(dist2), PatDp(dist2), and wild-type embryos were identified at $15.5 \mathrm{dpc}$ by typing for the marker D2Mit226 (Williamson et al. 1995). The MatDp(dist2) and PatDp(dist2) embryos were used to establish the imprinting status of the FANTOM2 clones mapping to the Gnas cluster.

\section{Strand-Specific RT-PCR}

Poly(A) ${ }^{+}$RNA was extracted from MatDp(dist2) and PatDp(dist2) embryos using the FastTrack 2.0 mRNA isolation kit (Invitrogen) and the RNA was treated with RNase-free DNase I using the Message Clean kit (GenHunter Corporation). Primers to amplify cDNAs expressed from the Gnas cluster were designed from the BAC sequence (accession no. AL593857, clone number RP23-439H2) and were supplied by SigmaGenosys (Cambridge, UK). The relative positions of the primers are shown in Figure 1, and their sequence is provided in a Supplementary Table 2.

Strand-specific RT-PCR was performed as described by Wroe et al. (2000). Each RNA sample was reverse transcribed in the presence and absence of reverse transcriptase. Strandspecific primers and $1 \mu \mathrm{g}$ of oligo $(\mathrm{dA})_{80}$ [Genosys] were added to $0.15 \mu \mathrm{g}$ of poly $(\mathrm{A})^{+} \mathrm{RNA}$, and the mixture was heated at $70^{\circ} \mathrm{C}$ for $10 \mathrm{~min}$; the $(\mathrm{dA}) 80$ oligonucleotide was added to all samples, except those with oligo(dT)15 primer (Promega), to trap any oligo(dT) that might have co-purified with the poly $(A)^{+}$RNA (Nguyen et al. 1995). First-strand cDNA was synthesized at $50^{\circ} \mathrm{C}$ for $50 \mathrm{~min}$ by using either sense or antisense primers with Superscript II (200 units; Life Technologies). The enzyme was inactivated at $80^{\circ} \mathrm{C}$ for $45 \mathrm{~min}$. Firststrand cDNA was amplified by PCR, consisting of 20-30 cycles (as described in the legend of Fig. 2) of 1 min each at $94^{\circ} \mathrm{C}$, $55^{\circ} \mathrm{C}$, and $72^{\circ} \mathrm{C}$ by using Thermoprime Plus DNA polymerase (Advanced Biotechnologies). Extensor Long Master Mix (Advanced Biotechnologies) was used to amplify FANTOM cDNAs 3, 8, and 9. cDNA (200ng) and each primer (300 nM) were used in a 50- $\mu \mathrm{L}$ reaction and amplified using the following conditions: $94^{\circ} \mathrm{C}$ for $10 \mathrm{sec}, 62^{\circ} \mathrm{C}$ for $30 \mathrm{sec}$, and $68^{\circ} \mathrm{C}$ for $2 \mathrm{~min}$ for 10 cycles followed by 20 cycles at $94^{\circ} \mathrm{C}$ for $20 \mathrm{sec}$, $60^{\circ} \mathrm{C}$ for $30 \mathrm{sec}$, and $68^{\circ} \mathrm{C}$ for $2 \mathrm{~min}$.

\section{Southern Hybridization}

DNA was transferred onto charged nylon membranes (Hybond $\mathrm{N}^{+}$; Amersham Pharmacia) by alkaline transfer. PCR products were radiolabeled with $25 \mu \mathrm{Ci}$ of $\left[-{ }^{32} \mathrm{P}\right] \mathrm{dCTP}(\mathrm{NEN})$ using Megaprime (Amersham Pharmacia). The Southern filters were hybridized using Church and Gilbert hybridization buffers (Church and Gilbert 1984). The products of F3 (f) and F4 (g) were probed with PCR amplified BAC DNA (clone number RP23-439H2) using forward and reverse primers for EST AA955518, and the PCR products of F8 and F9 (j) were probed with the PCR amplified BAC DNA using primers FAN 8F and FAN 8R.

\section{ACKNOWLEDGMENTS}

The authors thank Dr. Gavin Kelsey of the Babraham Institute, Cambridge, UK, and Professor David Hume of the CRC for Chronic Inflammatory Disease, IMB, The University of QLD, Australia, for invaluable discussions and advice. We thank J. Skinner of MRC Harwell for assistance with primer design and for assistance with isolating BACs for sequencing through the MRC UK Mouse Genome Sequencing Programme; and C.V. Beechey, E. Prescott, and J. McNaughton of

\section{Genome Research


MRC Harwell for mouse resources. C.W. is funded by the CRC for Chronic Inflammatory Diseases, IMB, The University of QLD, Australia. All animal studies were carried out under the guidance issued by the Medical Research Council in "Responsibility in the Use of Animals for Medical Research" (July 1993) and Home Office Project License No. 30/1518.

\section{REFERENCES}

Bonthron, D.T., Hayward, B.E., Moran, V., and Strain, L. 2000 Characterization of TH1 and CTSZ, two non-imprinted genes downstream of GNAS1 in chromosome 20q13. Hum. Genet. 107: 165-175.

Cattanach, B.M. and Kirk, M. 1985. Differential activity of maternally and paternally derived chromosome regions in mice. Nature 315: $496-498$.

Cattanach, B.M., Peters, J., Ball, S., and Rasberry, C. 2000. Two imprinted gene mutations: Three phenotypes. Hum. Mol. Genet. 9: 2263-2273.

Church, G.M. and Gilbert, W. 1984. Genomic sequencing. Proc. Natl. Acad. Sci. 81: 1991-1995.

Crawford, J.A., Mutchler, K.J., Sullivan, B.E., Lanigan, T.M., Clark, M.S., and Russo, A.F. 1993. Neural expression of a novel alternatively spliced and polyadenylated Gs $\alpha$ transcript. J. Biol. Chem. 268: 9879-9885.

The FANTOM Consortium and the RIKEN Genome Exploration Research Group Phase I \& II Team. 2002. Analysis of the mouse transcriptome based on functional annotation of 60,770 full-length cDNAs. Nature 420: 563-573.

Hayward, B.E. and Bonthron, D.T. 2000. An imprinted antisense transcript at the human GNAS1 locus. Hum. Mol. Genet. 9: 835-841.

Hayward, B.E., Kamiya, M., Strain, L., Moran, V., Campbell, R., Hayashizaki, Y., and Bonthron, D.T. 1998. The human GNAS1 gene is imprinted and encodes distinct paternally and biallelically expressed G proteins. Proc. Natl. Acad. Sci. 95: $10038-10043$.

Kelsey, G., Bodle, D., Miller, H.J., Beechey, C.V., Coombes, C., Peters, J., and Williamson, C.M. 1999. Identification of imprinted loci by methylation-sensitive representational difference analysis: Application to mouse distal chromosome 2. Genomics 62: 129-138.

Lee, J.T. 2000. Disruption of imprinted X inactivation by parent-of-origin effects at Tsix. Cell 103: 17-27.

Li, T., Vu, T.H., Zeng, Z.L., Nguyen, B.T., Hayward, B.E., Bonthron, D.T., Hu, J.F., and Hoffman, A.R. 2000. Tissue-specific expression of antisense and sense transcripts at the imprinted Gnas locus. Genomics 69: 295-304.

Liu, J., Yu, S., Litman, D., Chen, W., and Weinstein, L.S. 2000. Identification of a methylation imprint mark within the mouse Gnas locus. Mol. Cell. Biol. 20: 5808-5817.
Lyle, R., Watanabe, D., te Vruchte, D., Lerchner, W., Smrzka, O.W., Wutz, A., Schageman, J., Hahner, L., Davies, C., and Barlow, D.P. 2000. The imprinted antisense RNA at the Igf2r locus overlaps but does not imprint Mas1. Nat. Genet. 25: 19-21.

Nguyen, C., Rocha, D., Granjeaud, S., Baldit, M., Bernard, K., Naquet, P., and Jordan, B.R. 1995. Differential gene expression in the murine thymus assayed by quantitative hybridization of arrayed cDNA clones. Genomics 29: 207-216.

Peters, J., Wroe, S.F., Wells, C.A., Miller, H.J., Bodle, D., Beechey, C.V., Williamson, C.M., and Kelsey, G. 1999. A cluster of oppositely imprinted transcripts at the Gnas locus in the distal imprinting region of mouse chromosome 2. Proc. Natl. Acad. Sci. 96: $3830-3835$.

Searle, A.G. and Beechey, C.V. 1978. Complementation studies with mouse translocations. Cytogenet. Cell. Genet. 20: 282-303.

Skinner, J.S., Cattanach, B.M., and Peters, J. 2002. The imprinted oedematous-small mutation on mouse chromosome 2 identifies new roles for Gnas and Gnasxl in development. Genomics 80: $373-375$.

Sleutels, F., Zwart, R., and Barlow, D.P. 2002. The non-coding Air RNA is required for silencing autosomal imprinted genes. Nature 415: $810-813$.

Williamson, C.M., Miller, H., Beechey, C. and Peters, J. 1995. Microsatellite marker D2Mit226 for the identification of duplication/deficient mice for the distal region of Chr 2. Mouse Genome 93: 860.

Williamson, C.M., Schofield, J., Dutton, E.R., Seymour, A., Beechey, C.V., Edwards, Y.H., and Peters, J. 1996. Glomerular-specific imprinting of the mouse Gs $\alpha$ gene: How does this relate to hormone resistance in albright hereditary osteodystrophy? Genomics 36: 280-287.

Williamson, C.M., Skinner, J.A., Kelsey, G., and Peters, J. 2002. Alternative non-coding splice variants of Nespas, an imprinted gene antisense to Nesp in the Gnas imprinting cluster. Mamm. Genome 13: 74-79.

Wroe, S.F., Kelsey, G., Skinner, J.A., Bodle, D., Ball, S.T., Beechey, C.V., Peters, J., and Williamson, C.M. 2000. An imprinted transcript, antisense to Nesp, adds complexity to the cluster of imprinted genes at the mouse Gnas locus. Proc. Natl. Acad. Sci. 97: 3342-3346.

Wutz, A., Smrzka, O.W., Schweifer, N., Schellander, K., Wagner, E.F., and Barlow, D.P. 1997. Imprinted expression of the Igf2r gene depends on an intronic CpG island. Nature 389: 745-749.

Yu, S., Yu, D., Lee, E., Eckhaus, M., Lee, R., Corria, Z., Accili, D., Westphal, H., and Weinstein, L.S. 1998. Variable and tissue-specific hormone resistance in heterotrimeric Gs protein $\alpha$-subunit (Gs $\alpha)$ knockout mice is due to tissue-specific imprinting of the Gs $\alpha$ gene. Proc. Natl. Acad. Sci. 95: 8715-8720.

Received November 5, 2002; accepted in revised form February 19, 2003. 


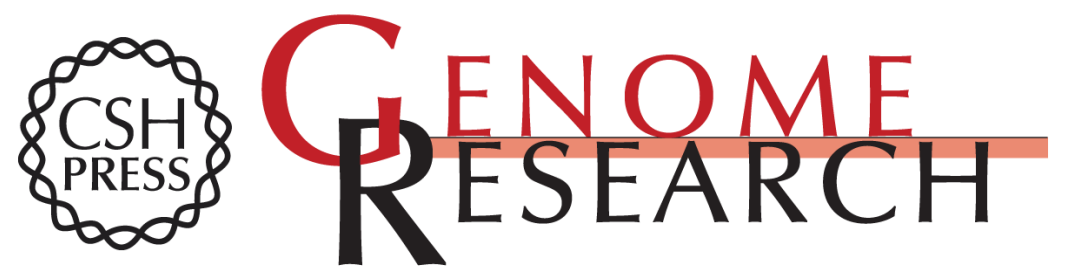

\section{A Comprehensive Transcript Map of the Mouse Gnas Imprinted Complex}

Rebecca Holmes, Christine Williamson, Jo Peters, et al.

Genome Res. 2003 13: 1410-1415

Access the most recent version at doi:10.1101/gr.955503

Supplemental Material

References

License

Email Alerting Service
http://genome.cshlp.org/content/suppl/2003/06/22/13.6b.1410.DC1

This article cites 24 articles, 7 of which can be accessed free at: http://genome.cshlp.org/content/13/6b/1410.full.html\#ref-list-1

Receive free email alerts when new articles cite this article - sign up in the box at the top right corner of the article or click here.

\section{Affordable, Accurate Sequencing.}

\section{gencove}

To subscribe to Genome Research go to: https://genome.cshlp.org/subscriptions 